\title{
Factors of IID on Trees
}

\author{
By Russell LyONS
}

\begin{abstract}
Classical ergodic theory for integer-group actions uses entropy as a complete invariant for isomorphism of IID (independent, identically distributed) processes (a.k.a. product measures). This theory holds for amenable groups as well. Despite recent spectacular progress of Bowen, the situation for non-amenable groups, including free groups, is still largely mysterious. We present some illustrative results and open questions on free groups, which are particularly interesting in combinatorics, statistical physics, and probability. Our results include bounds on minimum and maximum bisection for random cubic graphs that improve on all past bounds.
\end{abstract}

\section{$\S 1$. Introduction.}

Let $\Gamma$ be a group and $X$ and $Y$ be two sets on which $\Gamma$ acts. A map $\phi: X \rightarrow Y$ is called $\Gamma$-equivariant if $\phi$ intertwines the actions of $\Gamma$ :

$$
\phi(\gamma x)=\gamma(\phi(x)) \quad(\gamma \in \Gamma, x \in X)
$$

If $X$ and $Y$ are both measurable spaces, then a $\Gamma$-equivariant measurable $\phi$ is called a $\Gamma$-factor. Let $\mu$ be a measure on $X$. If $\phi$ is a $\Gamma$-factor, then the push-forward measure $\phi_{*} \mu$ is called a $\Gamma$-factor of $\mu$. The measure $\mu$ is $\Gamma$-invariant if

$$
\mu(\gamma B)=\mu(B) \quad(\gamma \in \Gamma, B \subseteq X \text { measurable })
$$

If $\nu$ is a measure on $Y$, then a $\nu$-a.e.-invertible $\Gamma$-factor $\phi$ such that $\nu=\phi_{*} \mu$ is called an isomorphism from $(X, \mu, \Gamma)$ to $(Y, \nu, \Gamma)$. Classical ergodic theory is concerned with the case that $\Gamma=\mathbb{Z}$. In probability theory, we often have that $X$ and $Y$ are product spaces of

2010 Mathematics Subject Classification. Primary 05C30, 05C70, 05C80, 37A35, 37A50, 60G10. Secondary 60G15.

Key words and phrases. Non-amenable groups, regular graphs, bisection, local rules, Ising, tail field. Research partially supported by Microsoft Research and NSF grant DMS-1007244. 
the form $\mathfrak{A}^{\Gamma}$ or, more generally, $\mathfrak{A}^{W}$, where $\mathfrak{A}$ is a measurable space, called the base, and $W$ is a countable set on which $\Gamma$ acts. Note that in this case, $\Gamma$ acts on $\mathfrak{A}^{W}$ by

$$
(\gamma \omega)(x):=\omega\left(\gamma^{-1} x\right) \quad\left(\omega \in \mathfrak{A}^{W}, x \in W, \gamma \in \Gamma\right) .
$$

When $\mathfrak{A}$ is finite, $\lambda$ is the uniform measure on $\mathfrak{A}$, and $\Gamma$ is denumerable, then $\left(\mathfrak{A}^{\Gamma}, \lambda^{\Gamma}, \Gamma\right)$ is called the $|\mathfrak{A}|$-shift over $\Gamma$. When $\mu$ and $\nu$ are more general product measures with finite base spaces and $\Gamma=\mathbb{Z}$, the problem of whether $\left(\mathfrak{A}^{\Gamma}, \mu, \Gamma\right)$ and $\left(\mathfrak{A}^{\Gamma}, \nu, \Gamma\right)$ are isomorphic is very old. It was solved through the introduction of entropy by Kolmogorov $(1958,1959)$ and Sinal (1959), and the work of Ornstein (1970a). In particular, the entropy of a $k$-shift is $\log k$. Factors play a key role in this and other aspects of ergodic theory.

The theory of entropy and its applications was extended to amenable groups by Ornstein and Weiss (1987). One important feature is that the entropy of a factor of an invariant probability measure $\mu$ is at most the entropy of $\mu$. Ornstein and Weiss (1987) noted that no reasonable definition of entropy on free groups of rank at least 2 has this property, since, as they showed, the 4 -shift is a factor of the 2-shift over such groups. Clearly the 2-shift is also a factor of the 4-shift. The problem whether the 2-shift and the 4-shift, for example, are isomorphic was finally solved by Bowen (2010), who introduced a notion of entropy for free-group actions that is invariant under isomorphism. His notion of entropy again assigns the value $\log k$ to a $k$-shift over any free group. Bowen's work is the analogue of that of Kolmogorov and Sina. . However, most of Ornstein theory remains terra incognita.

One reason, therefore, to study factors over free groups, and especially factors of product measures, is to understand how to extend Ornstein theory. Other reasons arise from questions in probability theory, combinatorics, and computer science, as well as the ergodic theory of equivalence relations. We shall discuss questions from most of these areas here, providing some results and highlighting some particularly interesting open questions.

Recent papers concerning factors (generally of IID processes or their continuous analogue, Poisson point processes) that involve a mix of probability and combinatorics include Holroyd and Peres (2003), Timár (2004), Ball (2005b), Holroyd, Pemantle, Peres, and Schramm (2009), Chatterjee, Peled, Peres, and Romik (2010), Soo (2010), Holroyd (2011), Holroyd, Lyons, and Soo (2011), Timár (2011), Mester (2011), Lyons and Nazarov (2011), Angel, Benjamini, Gurel-Gurevich, Meyerovitch, and Peled (2012), Csóka and Lippner (2012), Gamarnik and Sudan (2013), Csóka, Gerencsér, Harangi, and Virág (2015), Harangi and Virág (2015), Backhausz, Szegedy, and Virág (2015), Conley (2013), Kun (2013), Quas and Soo (2016), and Gurel-Gurevich and Peled (2013).

The utility of factors of IID processes on non-amenable groups has been shown in various ways. For example, see Popa (2006), Chifan and Ioana (2010), Houdayer (2012), 
Lyons (2013), Abért and Weiss (2013), and Kun (2013).

Because in many situations one has the natural Cayley graph of a free group, that is, a regular tree, one also is often interested in processes that are invariant under the full automorphism group of the tree and, similarly, in factors that are equivariant with respect to the full automorphism group. Thus, let $\mathbb{T}_{d}$ be a $d$-regular tree with $d \geq 3$. When $d$ is even, this is a Cayley graph of the free group $\mathbb{F}_{d / 2}$ on $d / 2$ generators. In all cases, it is a Cayley graph of the free product of $d$ copies of $\mathbb{Z}_{2}$.

Our greatest interest in this paper is $\operatorname{Aut}\left(\mathbb{T}_{d}\right)$-factors

$$
\phi:\left([0,1]^{\mathrm{V}\left(\mathbb{T}_{d}\right)}, \mathcal{L}^{\mathrm{V}\left(\mathbb{T}_{d}\right)}\right) \rightarrow\{0,1\}^{\mathrm{V}\left(\mathbb{T}_{d}\right)},
$$

where $\mathcal{L}$ is Lebesgue measure on $[0,1]$. We shall often leave off the prefix $\operatorname{Aut}\left(\mathbb{T}_{d}\right)$ from the word "factor". Since the domain space is product measure, or IID, such a $\phi$ is called a factor of IID, or FIID for short. The elements of the domain space are sometimes called labels. The push-forward measure $\phi_{*} \mathcal{L}^{\mathrm{V}\left(\mathbb{T}_{d}\right)}$ is also called an FIID. Under the same rubric we shall consider other product measures over either $\mathrm{V}\left(\mathbb{T}_{d}\right)$ or $\mathrm{E}\left(\mathbb{T}_{d}\right)$, with the codomain also being other product measurable spaces over either $\mathrm{V}\left(\mathbb{T}_{d}\right)$ or $\mathrm{E}\left(\mathbb{T}_{d}\right)$.

Extending the fundamental example of Ornstein and Weiss (1987), Ball (2005a) showed that the 4-shift is an $\operatorname{Aut}\left(\mathbb{T}_{d}\right)$-factor of the 2-shift, as is $\mathcal{L}^{\mathrm{V}\left(\mathbb{T}_{d}\right)}$. For this reason, it matters little which product measure is used as the domain of a factor.

Our contributions in this area are to exhibit weak* limits of FIID processes that are not themselves FIID; and to use FIID processes in order to improve on existing bounds for minimum and maximum bisection of random regular graphs. For example, Monien and Preis (2001) showed that random 3-regular graphs asymptotically have bisection width at most $1 / 6$, which we improve to 0.1623 .

\section{$\S 2$. Factors on Trees.}

Let o denote a fixed vertex, the root, of $\mathbb{T}_{d}$. There is a correspondence between $\operatorname{Aut}\left(\mathbb{T}_{d}\right)$-factors of IID, $\phi$, and spherically symmetric measurable functions $F:[0,1]^{\mathrm{V}\left(\mathbb{T}_{d}\right)} \rightarrow$ $\{0,1\}$, namely,

$$
F(\omega)=(\phi(\omega))(\mathbf{o}) \quad\left(\omega \in[0,1]^{\vee}\right)
$$

in one direction and

$$
(\phi(\omega))\left(\gamma^{-1} \mathbf{o}\right)=F(\gamma \omega) \quad\left(\omega \in[0,1]^{\vee}, \gamma \in \operatorname{Aut}\left(\mathbb{T}_{d}\right)\right)
$$

in the other. 
For a measurable space $\mathfrak{A}$, write $\pi_{x}: \mathfrak{A}^{\vee} \rightarrow \mathfrak{A}$ for the natural coordinate projections $(x \in \mathrm{V})$. For $K \subseteq \mathrm{V}$, write $\mathscr{F}(K)$ for the $\sigma$-field on $\mathfrak{A}^{\mathrm{V}}$ generated the maps $\pi_{x}$ for $x \in K$. Let $B_{r}:=B_{r}(\mathbf{o})$ be the graph induced on the set of vertices within graph distance $r$ of $\mathbf{o}$. We may approximate $F$ as in (2.1) by spherically symmetric measurable maps $F_{r}:[0,1]^{\mathrm{V}\left(B_{r}(\mathbf{o})\right)} \rightarrow\{0,1\}$ that converge to $F$ a.s. For example, the conditional expectations $F_{r}:=\mathbb{E}\left[F \mid \mathscr{F}\left(B_{r}(\mathbf{o})\right)\right]$ converge to $F$ by Lévy's 0-1 Law. These maps $F_{r}$ determine FIIDs $\phi_{r}$ via (2.2), called block factors of IID or local rules. We have that $\phi_{r}$ converges to $\phi$ a.s. (in the product topology) and therefore $\left(\phi_{r}\right)_{*} \mathcal{L}^{\vee}$ converges to $\phi_{*} \mathcal{L}^{\vee}$ in Ornstein's $\bar{d}$-metric. This metric is defined as follows. Let $\mu_{1}$ and $\mu_{2}$ be two $\Gamma$-invariant probability measures on $\mathfrak{A}^{W}$, where $\Gamma$ acts quasi-transitively on $W$ and $\mathfrak{A}$ is a finite set. Let $W^{\prime}$ be a section of $\Gamma \backslash W$. Then

$\bar{d}\left(\mu_{1}, \mu_{2}\right):=\min \left\{\sum_{w \in W^{\prime}} \mathbb{P}\left[X_{1}(w) \neq X_{2}(w)\right] ; X_{1} \sim \mu_{1}, X_{2} \sim \mu_{2},\left(X_{1}, X_{2}\right)\right.$ is $\Gamma$-invariant $\}$.

The tail $\sigma$-field is defined to be $\bigcap_{r} \mathscr{F}\left(\mathrm{V} \backslash B_{r}\right)$. For $x \in \mathrm{V}$, let $D_{x}$ denote the set of vertices separated from o by $x$. If $\left(x_{1}, x_{2}, \ldots\right)$ is a simple path of vertices in $\mathbb{T}_{d}$, the corresponding 1-ended tail $\sigma$-field is $\bigcap_{n} \mathscr{F}\left(D\left(x_{n}\right)\right)$. Let $\operatorname{Aut}_{+}\left(\mathbb{T}_{d}\right)$ denote the parity-preserving subgroup of $\operatorname{Aut}\left(\mathbb{T}_{d}\right)$, i.e., Aut $\left(\mathbb{T}_{d}\right):=\left\{\gamma \in \operatorname{Aut}\left(\mathbb{T}_{d}\right) ; \forall x \in \mathrm{V}\left(\mathbb{T}_{d}\right) d(x, \gamma x) \in 2 \mathbb{N}\right\}$, where $d(x, y)$ is the graph distance between $x$ and $y$. Every $\operatorname{Aut}\left(\mathbb{T}_{d}\right)$-invariant $\operatorname{Aut}\left(\mathbb{T}_{d}\right)$-ergodic probability measure on $\mathfrak{A}^{\vee}$ is an equal mixture of two Aut $+\left(\mathbb{T}_{d}\right)$-invariant Aut $+\left(\mathbb{T}_{d}\right)$-ergodic probability measures, and the latter have trivial 1-ended tail $\sigma$-fields, as shown by Pemantle (1992). By virtue of being Aut $+\left(\mathbb{T}_{d}\right)$-ergodic, every FIID has trivial 1-ended tails.

A probability measure $\mu$ on $\mathfrak{A}^{\vee}$ is called m-dependent if $\mathscr{F}\left(K_{1}\right), \ldots, \mathscr{F}\left(K_{p}\right)$ are independent whenever the sets $K_{i}$ are pairwise separated by graph distance $>m$. We say that $\mu$ is finitely dependent if it is $m$-dependent for some $m<\infty$. For example, a block FIID that depends on the ball of radius $r$ is $2 r$-dependent.

According to the Kolmogorov 0-1 Law, the tail $\sigma$-field is trivial for every IID probability measure. Reasoning similar to its proof shows the second of the following implications for $\operatorname{Aut}\left(\mathbb{T}_{d}\right)$-invariant processes:

block FIID $\Longrightarrow$ finitely dependent $\Longrightarrow$ trivial tail.

It is open whether finitely dependent implies FIID and whether trivial tail implies FIID. These questions are resolved on $\mathbb{Z}$ : finitely dependent implies FIID by using the VWB condition of Ornstein (1974) and trivial tail does not imply FIID (even for finite $\mathfrak{A}$ ) by Ornstein (1973) and Kalikow (1982). It is also known that for finite $\mathfrak{A}$, FIID implies trivial 1-ended tail $\sigma$-fields, as proved by Rohlin and Sinaŭ (1961). This latter implication is false 
for $\mathfrak{A}=[0,1]$. We note, however, that Smorodinsky (1971) proved that Gaussian processes on $\mathbb{Z}$ with trivial 1-ended tail are FIID.

The following question is due to Bowen (2013):

QUESTION 2.1. Is every FIID process isomorphic to an IID process?

Ornstein (1970b) proved this holds on $\mathbb{Z}$. It does not suffice on $\mathbb{T}_{d}$ to have factor maps each way, since this holds for the 2-shift and 4-shift, but these are not, by Bowen (2010), isomorphic. Note that Popa (2006) proved that there exist non-amenable groups where FIIDs are not necessarily isomorphic to IIDs.

Many of the above questions can be asked about invariant processes on non-amenable groups more generally, not just free groups.

QUESTION 2.2. Is every finitely dependent process an FIID?

This holds in the amenable case again by using the VWB condition, here defined by Adams (1992).

We now present an example of an FIID on $\mathbb{T}_{d}$ with finite $\mathfrak{A}$ whose tail $\sigma$-field is full (everything). Such examples on $\mathbb{Z}$ were given by Ornstein and Weiss (1975) (who proved that every process is isomorphic to one whose tail $\sigma$-field is full), Burton, Denker, and Smorodinsky (1996), and Burton and Steif (1997).

Proposition 2.3. There exists a unique Aut $\left(\mathbb{T}_{d}\right)$-invariant probability measure, $\mu^{\mathrm{pm}}$, on the set of perfect matchings of $\mathbb{T}_{d}$; it is an FIID whose tail $\sigma$-field is full.

Proof. Since the stabilizer $\Gamma$ of $\mathbf{o}$ in $\operatorname{Aut}\left(\mathbb{T}_{d}\right)$ acts transitively on the set of perfect matchings of $\mathbb{T}_{d}$, there is a unique $\Gamma$-invariant probability measure, $\mu^{\mathrm{pm}}$, on the set of perfect matchings. This measure is easy to construct by starting at o, choosing uniformly at random one of its $d$ incident edges to be in the matching, and then working outwards independently, where every time there is a choice between $d-1$ edges, they are equally likely to be in the matching. Using the independence, it is not hard to see that $\mu^{\mathrm{pm}}$ is actually $\operatorname{Aut}\left(\mathbb{T}_{d}\right)$-invariant. Although it is far from obvious, $\mu^{\mathrm{pm}}$ is an FIID, as shown by Lyons and Nazarov (2011).

To see that the tail is full, consider any event $A$ of perfect matchings and any radius $r \geq 0$. Let $A_{r}$ be the event consisting of all perfect matchings that agree with some element of $A$ when restricted to the complement of $\mathrm{E}\left(B_{r}(\mathbf{o})\right)$. We claim that $A=A_{r}$ for all $r$, which will imply that the tail of $\mu^{\mathrm{pm}}$ is full. We prove this by induction on $r$. It is clear that $A_{0}=A$. Now let $\omega \in A$ and $r \geq 0$. By definition, there exists some $\omega^{\prime} \in A_{r+1}$ that agrees with $\omega$ outside $B_{r+1}(\mathbf{o})$. Consider an edge $e \in B_{r+1}(\mathbf{o}) \backslash B_{r}(\mathbf{o})$. Let $F$ be the set of $d-1$ edges incident to $e$ that do not lie in $B_{r+1}(\mathbf{o})$. Since $\omega(e)=1$ iff $\omega(f)=0$ for 
all $f \in F$, and likewise for $\omega^{\prime}$, it follows that $\omega(e)=\omega^{\prime}(e)$, whence that $\omega$ agrees with $\omega^{\prime}$ outside $B_{r}(\mathbf{o})$, i.e., that $\omega^{\prime} \in A_{r}$. Therefore, $A=A_{r}$ implies that $A=A_{r+1}$, which completes the induction.

For similar reasons, there is a unique $\operatorname{Aut}\left(\mathbb{T}_{d}\right)$-invariant probability measure, $\mu^{\text {col }}$, on the set of proper $d$-colorings of $\mathrm{E}\left(\mathbb{T}_{d}\right)$. This measure is again easy to construct by working outwards from o. Proper $d$-colorings can also be regarded as Cayley diagrams of the free product, $\mathbb{Z}_{2}^{* d}$, of $d$ copies of $\mathbb{Z}_{2}$.

Question 2.4. Is $\mu^{\mathrm{col}}$ an FIID?

This is open. A positive answer would imply that the set of $\operatorname{Aut}\left(\mathbb{T}_{d}\right)$-factors is equal to the set of $\operatorname{Aut}\left(\mathbb{T}_{d}\right)$-invariant $\mathbb{Z}_{2}^{* d}$-factors. Note that every $\mathbb{Z}_{2}^{* d}$-invariant probability measure induces an $\operatorname{Aut}\left(\mathbb{T}_{d}\right)$-invariant probability measure by averaging with respect to the stabilizer of $\mathbf{o}$ in $\operatorname{Aut}\left(\mathbb{T}_{d}\right)$.

Let $1, \ldots, d$ be the $d$ colors we use. It is also open whether $\mu^{\mathrm{col}}=\phi_{*} \mu^{\mathrm{pm}}$ for some $\operatorname{Aut}\left(\mathbb{T}_{d}\right)$-factor $\phi$ that colors every edge in the perfect matching by color 1 , i.e., $(\phi(\omega))(e)=$ 1 for all $e$ with $\omega(e)=1$.

We mention a partial result towards answering Question 2.4. A proper $d$-coloring is the same as a list $\left(P_{1}, \ldots, P_{d}\right)$ of $d$ disjoint perfect matchings. It is possible to obtain as an FIID a probability measure on lists $\left(P_{1}, \ldots, P_{d-2},\left\{Q_{1}, Q_{2}\right\}\right)$, where $P_{i}$ and $Q_{j}$ are disjoint perfect matchings, but $\left\{Q_{1}, Q_{2}\right\}$ is unordered. Indeed, choose $P_{1}$ via the FIID $\mu^{\mathrm{pm}}$. Note that $([0,1], \mathcal{L})$ is isomorphic to $\left([0,1]^{\mathbb{N}}, \mathcal{L}^{\mathbb{N}}\right)$, so that when we create $P_{1}$ as an FIID, we may use only the first coordinates of the labels, reserving the later coordinates for further use. Deleting the edges of $P_{1}$ decomposes $\mathbb{T}_{d}$ into a forest of copies of $\mathbb{T}_{d-1}$. Provided $d-1 \geq 3$, we may choose perfect matchings in each copy by using the second coordinates of the labels and let $P_{2}$ be their union. This procedure may be continued until we are left with trees of degree 2. Each such tree is decomposed uniquely as a set of 2 perfect matchings. We must decide, given a perfect matching $P$ of a tree $T$ of degree 2 and a perfect matching $P^{\prime}$ of another tree $T^{\prime}$ of degree 2, whether $P$ and $P^{\prime}$ belong to the same $Q_{i}$ or not. In order to make this decision for all perfect matchings and all trees, it suffices to make the decision for pairs of trees that are at distance 1 from each other in $\mathbb{T}_{d}$. In such a case, there is a unique edge $e$ that is incident to both trees. Let $e_{1}$ and $e_{2}$ be the two edges in $T$ that are adjacent to $e$ and $e_{1}^{\prime}$ and $e_{2}^{\prime}$ be the two edges in $T^{\prime}$ that are adjacent to $e$. Let $U_{i}$ and $U_{i}^{\prime}$ be the corresponding labels of these edges $(i=1,2)$. Then let the perfect matching containing $e_{1}$ belong to the same $Q_{i}$ as the perfect matching containing $e_{1}^{\prime}$ iff $\left(U_{1}-U_{2}\right)\left(U_{1}^{\prime}-U_{2}^{\prime}\right)>0$. 


\section{$\S 3$. Tree-Indexed Markov Chains and Ising Measures.}

Next we consider the simplest types of invariant processes after IID, namely, 2-state symmetric $\mathbb{T}_{d}$-indexed Markov chains. Let $|\theta| \leq 1$ and consider the transition matrix

$$
\left(\begin{array}{cc}
\frac{1+\theta}{2} & \frac{1-\theta}{2} \\
\frac{1-\theta}{2} & \frac{1+\theta}{2}
\end{array}\right) \text {. }
$$

For $\theta \geq 0$, another way to think of this transition matrix, which explains this parametrization, is to keep the same state with probability $\theta$ and to choose uniformly among the two states independently of the current state with probability $1-\theta$. For $\theta \leq 0$, the interpretation is slightly different: change to the opposite state with probability $|\theta|$ and to choose uniformly among the two states independently of the current state with probability $1-|\theta|$. The tree-indexed Markov chain $\mu_{\theta}^{\mathrm{mc}}$ is obtained by assigning to the root one of the 2 states with equal probability, then proceeding to the neighbors of the root by using an independent transition from the above matrix, etc. When the two states are \pm 1 , this is known as the free Ising measure on $\mathbb{T}_{d}$, ferromagnetic when $\theta \geq 0$. In this case, the states are known as spins. We shall use this terminology for convenience.

The description of $\mu_{\theta}^{\mathrm{mc}}$ does not make it apparent that $\mu_{\theta}^{\mathrm{mc}}$ is an invariant measure, but it is not hard to check that it is indeed invariant. However, an important alternative description makes this invariance obvious. Namely, consider the clusters of Bernoulli $(|\theta|)$ bond percolation on $\mathbb{T}_{d}$. If $\theta \geq 0$, then for each cluster, assign all vertices the same spin, with probability $1 / 2$ for each spin, independently for different clusters. If $\theta \leq 0$, then assign each cluster one of its two proper \pm 1 -colorings, with probability $1 / 2$ each, independently for different clusters. It is easy to see that this gives $\mu_{\theta}^{\mathrm{mc}}$.

It is known that $\mu_{\theta}^{\mathrm{mc}}$ has a trivial tail iff $|\theta| \leq 1 / \sqrt{d-1}$. It is also known that $\mu_{\theta}^{\mathrm{mc}}$ is an FIID if $|\theta| \leq 1 /(d-1)$, but is not an FIID if $|\theta|>1 / \sqrt{d-1}$. It is open whether $\mu_{\theta}^{\mathrm{mc}}$ is an FIID for $1 /(d-1)<|\theta| \leq 1 / \sqrt{d-1}$. It is also open whether there is a critical $\theta_{0}$ such that $\mu_{\theta}^{\mathrm{mc}}$ is an FIID for $0 \leq \theta<\theta_{0}$ and not an FIID for $\theta_{0}<\theta \leq 1$; the analogous question is also open for $\theta<0$. The history of the result for tail triviality is reviewed in Sec. 2.2 of Evans, Kenyon, Peres, and Schulman (2000).

The fact that $\mu_{\theta}^{\mathrm{mc}}$ is an FIID for $|\theta| \leq 1 /(d-1)$ is easy to see: In this regime, all $|\theta|$-clusters are finite a.s. Let $U(e)$ and $U_{i}(x)$ be IID uniform $[0,1]$ random variables for $e \in \mathrm{E}\left(\mathbb{T}_{d}\right), i \in\{1,2\}$, and $x \in \mathrm{V}\left(\mathbb{T}_{d}\right)$. Choose the $|\theta|$-clusters by using the edges with $U(e) \leq|\theta|$. Given a cluster $C$, let its vertex with the minimum $U_{1}(x)$ be $x_{C}$ and let the spins in $C$ equal $\operatorname{sgn}\left(U_{2}\left(x_{C}\right)-1 / 2\right)$ if $\theta \geq 0$, while if $\theta<0$, let the spins in $C$ equal the proper \pm 1 -coloring whose spin at $x_{C}$ equals sgn $\left(U_{2}\left(x_{C}\right)-1 / 2\right)$.

In unpublished work, this author and later Lewis Bowen gave values $\theta_{d}$ such that for $|\theta|>\theta_{d}$, the measure $\mu_{d}^{\mathrm{mc}}$ is not an FIID. This was improved to $\theta_{d}=1 / \sqrt{d-1}$ by Sly 
(2009), but his proof was not published. We give that proof here because we shall adapt it to prove other results as well. This value of $\theta_{d}$ can also be established by using a result of Backhausz, Szegedy, and Virág (2015), which characterizes the rate of decay of the correlation of $\sigma(\mathbf{o})$ and $\sigma(x)$ as the distance between $\mathbf{o}$ and $x$ tends to infinity, where $\sigma$ is any FIID whose values at the vertices are real valued and square integrable. In particular, the correlation is at most $(n(d-2) / d+1) /(d-1)^{n / 2}$ in absolute value when the distance is $n$. Of course, this holds as well for weak* limits of FIID processes. In particular, weak* limits of FIID are strongly mixing, while on $\mathbb{Z}$, they need not even be ergodic. Note that $\mu_{\theta}^{\mathrm{mc}}$ has trivial 1-ended tails for all $|\theta|<1$.

The following is at the heart of Sly's proof, with the last observation about $\bar{d}_{2}$-closure due to this author and Peres in 2013. Here, given two invariant probability measures $\mu_{1}$ and $\mu_{2}$ on $\mathbb{R}^{\mathrm{V}}$, we define

$\bar{d}_{2}\left(\mu_{1}, \mu_{2}\right):=\min \left\{\mathbb{E}\left[\left|X_{1}(o)-X_{2}(o)\right|^{2}\right]^{1 / 2} ; X_{1} \sim \mu_{1}, X_{2} \sim \mu_{2},\left(X_{1}, X_{2}\right)\right.$ is $\Gamma$-invariant $\}$.

Note that FIID processes whose 1-dimensional marginals have finite second moments are $\bar{d}_{2}$-limits of block factors.

THEOREM 3.1. Let $G$ be a graph for which there is some unimodular group $\Gamma$ of automorphisms that acts transitively on $\mathrm{V}(G)$. Let $o \in \mathrm{V}(G)$. Write $S_{n}$ for the set of vertices at distance $n$ from o. Suppose that $x \mapsto \sigma(x)(x \in \mathrm{V}(G))$ is a $\Gamma$-invariant process with law $\mu$ on $\mathbb{R}^{\mathrm{V}(G)}$. Assume that $0<\operatorname{Var}(\sigma(o))<\infty$. Define $\boldsymbol{\Sigma}_{n}:=\sum_{x \in S_{n}} \sigma(x)$. If $\lim _{n \rightarrow \infty} \operatorname{Var}\left(\boldsymbol{\Sigma}_{n}\right) /\left|S_{n}\right|=\infty$ and $\limsup _{n \rightarrow \infty}\left|\operatorname{Corr}\left(\sigma(o), \boldsymbol{\Sigma}_{n}\right)\right|>0$, then $\mu$ is not a $\Gamma$ equivariant FIID, nor is $\mu$ in the $\bar{d}_{2}$-closure of the finitely dependent processes.

Note that the condition $\lim \sup _{n \rightarrow \infty}\left|\operatorname{Corr}\left(\sigma(o), \boldsymbol{\Sigma}_{n}\right)\right|>0$ alone implies that $\mu$ has a non-trivial tail.

Proof. Without loss of generality, we may assume that $\mathbb{E}[\sigma(o)]=0$ and that $\mathrm{SD}(\sigma(o))=1$. We shall show that if $\lim _{n \rightarrow \infty} \operatorname{Var}\left(\boldsymbol{\Sigma}_{n}\right) /\left|S_{n}\right|=\infty$ and $\mu$ lies in the $\bar{d}_{2}$-closure of the finitely dependent processes, then $\lim _{n \rightarrow \infty} \operatorname{Corr}\left(\sigma(o), \boldsymbol{\Sigma}_{n}\right)=0$.

Let $\epsilon>0$. Choose an invariant process $(X, Y)$ on $\mathbb{R}^{\vee} \times \mathbb{R}^{\vee}$ such that $X \sim \mu, Y$ is finitely dependent, and $\mathrm{SD}(X(o)-Y(o))<\epsilon$. For simplicity of notation, we take $X=\sigma$. Write $\boldsymbol{\Sigma}_{n}^{Y}:=\sum_{x \in S_{n}} Y(x)$. By the Mass-Transport Principle, we have that $\mathbb{E}\left[Y(o) \boldsymbol{\Sigma}_{n}\right]=$ $\mathbb{E}\left[\sigma(o) \boldsymbol{\Sigma}_{n}^{Y}\right]$. By finite dependence, we have $\operatorname{Var}\left(\boldsymbol{\Sigma}_{n}^{Y}\right)=O\left(\left|S_{n}\right|\right)=o\left(\operatorname{Var}\left(\boldsymbol{\Sigma}_{n}\right)\right)$, whence

$$
\mathbb{E}\left[Y(o) \boldsymbol{\Sigma}_{n} / \mathrm{SD}\left(\boldsymbol{\Sigma}_{n}\right)\right]=\mathbb{E}\left[\sigma(o) \boldsymbol{\Sigma}_{n}^{Y} / \mathrm{SD}\left(\boldsymbol{\Sigma}_{n}\right)\right] \rightarrow 0
$$

as $n \rightarrow \infty$. By the Cauchy-Schwarz inequality, we have

$$
\left|\mathbb{E}\left[Y(o) \boldsymbol{\Sigma}_{n} / \mathrm{SD}\left(\boldsymbol{\Sigma}_{n}\right)\right]-\mathbb{E}\left[\sigma(o) \boldsymbol{\Sigma}_{n} / \mathrm{SD}\left(\boldsymbol{\Sigma}_{n}\right)\right]\right| \leq \operatorname{SD}(Y(o)-\sigma(o))<\epsilon .
$$


Taking $n \rightarrow \infty$, it follows that

$$
\limsup _{n \rightarrow \infty}\left|\operatorname{Corr}\left(\sigma(o), \boldsymbol{\Sigma}_{n}\right)\right|=\limsup _{n \rightarrow \infty}\left|\mathbb{E}\left[\sigma(o) \boldsymbol{\Sigma}_{n} / \operatorname{SD}\left(\boldsymbol{\Sigma}_{n}\right)\right]\right|<\epsilon
$$

as desired.

The following is Sly's result.

Corollary 3.2. For $|\theta|>1 / \sqrt{d-1}$, the $\mathbb{T}_{d}$-indexed Markov chain $\mu_{\theta}^{\mathrm{mc}}$ is not an FIID. Proof. Let $|\theta|>1 / \sqrt{d-1}$ and $\sigma \sim \mu_{\theta}^{\mathrm{mc}}$. We verify the conditions of Theorem 3.1. Note that

$$
\operatorname{Corr}(\sigma(x), \sigma(y))=\mathbb{E}[\sigma(x) \sigma(y)]=\theta^{n}
$$

when $x$ and $y$ are at distance $n$ from each other. Also, $\left|S_{n}\right|=d(d-1)^{n-1}$. Therefore, $\mathbb{E}\left[\sigma(\mathbf{o}) \boldsymbol{\Sigma}_{n}\right]=\left|S_{n}\right| \theta^{n}$ and

$$
\operatorname{Var}\left(\boldsymbol{\Sigma}_{n}\right) /\left|S_{n}\right|=1+\sum_{k=1}^{n-1}(d-2)(d-1)^{k-1} \theta^{2 k}+(d-1)^{n} \theta^{2 n} \sim c\left|S_{n}\right| \theta^{2 n}
$$

for some constant $c$ as $n \rightarrow \infty$. Hence the conditions of Theorem 3.1 follow.

It is open whether discrete FIID processes are closed in the $\bar{d}$-topology, as they are on $\mathbb{Z}$ (see Ornstein (1974)). We shall use Theorem 3.1 to show that the class of FIID processes is not closed in the weak* topology, as is easy to show on $\mathbb{Z}$. This was also shown independently by Harangi and Virág (2015) on all infinite finitely generated groups, but their proof does not show the same for discrete processes.

Define $\rho_{d}:=2 \sqrt{d-1} / d$. The spectrum of the transition operator for simple random walk on $\mathbb{T}_{d}$ is the interval $\left[-\rho_{d}, \rho_{d}\right]$, as shown by Kesten (1959). Let $\sigma$ be the Gaussian wave function of Csóka, Gerencsér, Harangi, and Virág (2015) with eigenvalue $\rho$ for the transition operator; this is a centered Gaussian field on $\mathbb{T}_{d}$ whose covariances satisfy the recurrence

$$
c_{0}=1 ; c_{1}=\rho ;(d-1) c_{k+1}-d \rho c_{k}+c_{k-1}=0(k \geq 1),
$$

where $c_{k}$ is the covariance between each pair of vertices at distance $k$.

Corollary 3.3. There is a Gaussian process on $\mathbb{T}_{d}$ that is not an FIID but is a weak limit of FIID processes. There is a $\{0,1\}$-valued process that is not an FIID but is a weak* limit of FIID processes.

Proof. Let $\sigma$ be the Gaussian wave function with eigenvalue $\rho_{d}$ for the transition operator; Csóka, Gerencsér, Harangi, and Virág (2015) show that $\sigma$ is a weak* limit of FIIDs. Induction shows that

$$
\operatorname{Corr}(\sigma(x), \sigma(y))=(n(d-2) / d+1)(d-1)^{-n / 2}
$$


for $x$ and $y$ at distance $n$. Therefore, $\mathbb{E}\left[\sigma(\mathbf{o}) \boldsymbol{\Sigma}_{n}\right] \sim c n\left|S_{n}\right|^{1 / 2}$ and $\operatorname{Var}\left(\boldsymbol{\Sigma}_{n}\right) /\left|S_{n}\right| \sim c^{\prime} n^{2}$ for some positive constants $c$ and $c^{\prime}$ as $n \rightarrow \infty$. Hence the conditions of Theorem 3.1 follow.

Now let $\tau:=\operatorname{sgn} \sigma$. Since $\sigma$ is a weak ${ }^{*}$ limit of FIIDs, so is $\tau$. Note that there exist positive constants $c_{1}$ and $c_{2}$ such that if $Z_{1}$ and $Z_{2}$ are jointly normal random variables, then

$$
c_{1}\left|\operatorname{Corr}\left(\operatorname{sgn} Z_{1}, \operatorname{sgn} Z_{2}\right)\right| \leq\left|\operatorname{Corr}\left(Z_{1}, Z_{2}\right)\right| \leq c_{2}\left|\operatorname{Corr}\left(\operatorname{sgn} Z_{1}, \operatorname{sgn} Z_{2}\right)\right| .
$$

Hence the above calculations for $\sigma$ hold (up to bounded factors) for $\tau$ as well.

\section{$\S 4$. Edge Cuts in Finite Graphs.}

Weak* limits of FIID processes on $\mathbb{T}_{d}$ can be used to bound combinatorial quantities on random $d$-regular graphs or on $d$-regular graphs whose girth tends to infinity. More generally, they can be used on finite graphs whose random weak limit is $\mathbb{T}_{d}$. To explain this widely known idea, we first define "random weak limit" (for this restricted case).

For a vertex $x$ in a graph $G$, let $B_{r}(x ; G)$ denote the subgraph induced by the vertices in $G$ whose distance from $x$ is at most $r$. We consider this subgraph as rooted at $x$.

Let $\left\langle G_{n}\right\rangle$ be a sequence of finite graphs. For each $r \geq 1$, let $p_{n, r}$ denote the probability that a uniformly random vertex $x$ in $G_{n}$ satisfies the property that $B_{r}\left(x ; G_{n}\right)$ is rooted isomorphic to $B_{r}\left(\mathbf{o} ; \mathbb{T}_{d}\right)$, i.e., there is a graph isomorphism from $B_{r}\left(x ; G_{n}\right)$ to $B_{r}\left(\mathbf{o} ; \mathbb{T}_{d}\right)$ that sends $x$ to o. We say that the random weak limit of $\left\langle G_{n}\right\rangle$ is $\mathbb{T}_{d}$ if $\lim _{n \rightarrow \infty} p_{n, r}=1$ for every $r \geq 1$. It is evident that every sequence of $d$-regular graphs whose girth tends to infinity has this property. It is well known that if $G_{n}$ is a uniformly random $d$-regular graph on $n$ vertices (or, if $d$ is odd, on $2 n$ vertices), then also $\left\langle G_{n}\right\rangle$ has this property with probability 1. Other terms for this same concept are "Benjamini-Schramm convergence" and "local weak convergence".

Now, for the sake of concreteness, suppose that $\phi$ is a block FIID on $\mathbb{T}_{d}$ associated to the spherically symmetric measurable map $F:[0,1]^{\mathrm{V}\left(B_{r}\left(\mathbf{o} ; \mathbb{T}_{d}\right)\right)} \rightarrow\{0,1\}$. Given a graph $G$, one may assign independent uniform $[0,1]$ random variables to its vertices and then apply $F$ at every vertex $x$ for which $B_{r}(x ; G)$ is rooted isomorphic to $B_{r}\left(\mathbf{o} ; \mathbb{T}_{d}\right)$. At other vertices, assign the value 0 . In this way, we obtain a probability measure on $\{0,1\}^{\vee}(G)$ that is "close" to $\phi_{*} \mathcal{L}^{\mathrm{V}\left(\mathbb{T}_{d}\right)}$ when $G$ is "close" to $\mathbb{T}_{d}$. In particular, the expected number of vertices assigned the value 1 will be close to $\mathbb{P}[\phi(\bullet)(\mathbf{o})=1]$. Informally, we say that $\phi$ is emulated on $G$.

If we want to bound the number of vertices assigned 1 under some constraint on the set assigned 1, then exhibiting a random set obtained by emulating a block factor will 
help. Moreover, since every FIID is a weak* limit of block FIIDs, it generally suffices to find an FIID with the desired property on $\mathbb{T}_{d}$ and to calculate $\mathbb{P}[\phi(\bullet)(\mathbf{o})=1]$. Indeed, we may work with weak* limits of FIIDs.

We give two examples of this method that are inspired by Csóka, Gerencsér, Harangi, and Virág (2015). They were the first to use Gaussian factors for similar purposes.

A bisection of a finite graph $G$ is a subset $S \subset \mathrm{V}$ such that ||$S|-| \mathrm{V} \backslash S|| \leq 1$. In particular, if $|\mathrm{V}|$ is even, then $|S|=|\mathrm{V}| / 2$. The size of a bisection $S$, written size $(S)$, is the number of edges $E(S, \vee \backslash S)$ that join $S$ to $\vee \backslash S$. The problems of minimizing or maximizing the size of a bisection in a regular graph are known to be hard in various senses and are of interest in computer science; see Díaz, Do, Serna, and Wormald (2003). For a sequence of graphs $G_{n}$, define

$$
\operatorname{MinBi}:=\limsup _{n \rightarrow \infty} \min \left\{\operatorname{size}(S) /\left|\mathrm{V}\left(G_{n}\right)\right| ; S \text { is a bisection of } G_{n}\right\}
$$

and

$$
\text { MaxBi }:=\liminf _{n \rightarrow \infty} \max \left\{\operatorname{size}(S) /\left|\mathrm{V}\left(G_{n}\right)\right| ; S \text { is a bisection of } G_{n}\right\} .
$$

For random $d$-regular graphs, Bollobás (1988) proved that

$$
\operatorname{MinBi} \geq \frac{d}{4}-\frac{\sqrt{d \log 2}}{2}=\frac{d}{4}-0.416^{+} \sqrt{d}
$$

whereas Alon (1997) proved that

$$
\operatorname{MinBi} \leq \frac{d}{4}-\frac{3 \sqrt{d}}{32 \sqrt{2}}=\frac{d}{4}-0.0663^{-} \sqrt{d} .
$$

We improve the latter (upper) bound to $d / 4-0.32^{-} \sqrt{d}$. Still in the context of random $d$-regular graphs, Dembo, Montanari, and Sen (2015) establish the asymptotic values as $d \rightarrow \infty$

$$
\operatorname{MinBi}=\frac{d}{4}-\mathrm{P}_{*} \sqrt{\frac{d}{4}}+o(\sqrt{d})
$$

and

$$
\operatorname{MaxBi}=\frac{d}{4}+\mathrm{P}_{*} \sqrt{\frac{d}{4}}+o(\sqrt{d})
$$

where $\mathrm{P}_{*} \approx 0.7632$ is a certain known constant; for comparison with the previous bounds, note that $\mathrm{P}_{*} / 2 \approx 0.3816$. The best previous results on MinBi and MaxBi for random $d-$ regular graphs for specific $d$ can be found in Monien and Preis (2001), Díaz, Do, Serna, and Wormald (2003), and Díaz, Serna, and Wormald (2007). In the case of degrees $d=3,4$, we improve those results here, which were that a.s., $\mathrm{MinBi} \leq 1 / 6$ and $\mathrm{MaxBi} \geq 1.32595$ for $d=3$ and $\mathrm{MinBi} \leq 1 / 3$ and $\mathrm{MaxBi} \geq 5 / 3$ for $d=4$. We shall not actually need that our finite graphs be regular. 
THEOREM 4.1. Let $G_{n}$ be finite graphs whose random weak limit is $\mathbb{T}_{d}$ and whose average degree tends to $d \geq 3$. Then

$$
\operatorname{MinBi}<\frac{d}{2 \pi} \arccos \frac{2 \sqrt{d-1}}{d}<\frac{d}{4}-\frac{\sqrt{d}}{\pi}
$$

and

$$
\operatorname{MaxBi}>\frac{d}{2 \pi} \arccos \frac{-2 \sqrt{d-1}}{d}>\frac{d}{4}+\frac{\sqrt{d}}{\pi}
$$

For $d=3$, this gives

$$
\operatorname{MinBi}<\frac{3}{2 \pi} \arccos \sqrt{\frac{8}{9}}=0.1622602^{-}
$$

and

$$
\operatorname{MaxBi}>\frac{3}{2 \pi} \arccos \left(-\sqrt{\frac{8}{9}}\right)=1.3377398^{+} ;
$$

for $d=4$, it yields $\mathrm{MinBi}<1 / 3$ and $\mathrm{MaxBi}>5 / 3$.

Proof. We first make precise the connection of MinBi and MaxBi to weak* limits of FIID processes on $\mathbb{T}_{d}$. Let WF be the class of weak* limits $\phi$ of FIID processes on $\mathbb{T}_{d}$ with values $\phi(\bullet)(x) \in\{0,1\}$ for $x \in \mathrm{V}\left(\mathbb{T}_{d}\right)$ and with $\mathbb{P}[\phi(\bullet)(\mathbf{o})=1]=1 / 2$. Let MinBiFac be the infimum of $\mathbb{E}[|\{x \sim \mathbf{o} ; \phi(\bullet)(x) \neq \phi(\bullet)(\mathbf{o})\}|] / 2$ taken over $\phi \in \mathrm{WF}$; it is easily seen that this infimum is a minimum. Similarly, let MaxBiFac be the supremum of $\mathbb{E}[\mid\{x \sim \mathbf{o} ; \phi(\bullet)(x) \neq$ $\phi(\bullet)(\mathbf{o})\} \mid] / 2$ taken over the same $\phi \in \mathrm{WF}$. We claim that

$$
\operatorname{MinBi} \leq \operatorname{MinBiFac} \text { and } \mathrm{MaxBi} \geq \operatorname{MaxBiFac} .
$$

Indeed, let $\phi \in \mathrm{WF}$ and $\epsilon>0$. There exists a block FIID $\phi_{r} \in$ WF such that

$$
\left|\mathbb{E}[|\{x \sim \mathbf{o} ; \phi(\bullet)(x) \neq \phi(\bullet)(\mathbf{o})\}|]-\mathbb{E}\left[\left|\left\{x \sim \mathbf{o} ; \phi_{r}(\bullet)(x) \neq \phi_{r}(\bullet)(\mathbf{o})\right\}\right|\right]\right|<\epsilon / 2 .
$$

Now emulate $\phi_{r}$ on $G_{n}=\left(\mathrm{V}_{n}, \mathrm{E}_{n}\right)$. Let $S_{n} \subseteq \mathrm{V}_{n}$ be the subset of vertices assigned 1; this need not be a bisection, as we know only that $\mathbb{E}\left[\left|S_{n}\right|\right] /\left|\mathrm{V}_{n}\right| \rightarrow 1 / 2$ as $n \rightarrow \infty$. However, finite dependence of the block FIID $\phi_{r}$ implies that linear deviations from the mean of $\left|S_{n}\right|$ are exponentially unlikely as $n \rightarrow \infty$. Furthermore, $\mathbb{E}\left[\operatorname{size}\left(S_{n}\right)\right] /\left|\mathrm{V}_{n}\right|$ tends, as $n \rightarrow \infty$, to $\mathbb{E}\left[\left|\left\{x \sim \mathbf{o} ; \phi_{r}(\bullet)(x) \neq \phi_{r}(\bullet)(\mathbf{o})\right\}\right|\right] / 2$. We have similar exponentially fast convergence for this proportion, size $\left(S_{n}\right) /\left|\mathrm{V}_{n}\right|$. Thus, for large $n$, there exists $S_{n}$ such that

$$
\left|\frac{\left|S_{n}\right|}{\mathrm{V}_{n}}-\frac{1}{2}\right|<\frac{\epsilon}{8 d}
$$

and

$$
\left|\frac{\operatorname{size}\left(S_{n}\right)}{\left|\mathrm{V}_{n}\right|}-\frac{1}{2} \mathbb{E}\left[\left|\left\{x \sim \mathbf{o} ; \phi_{r}(\bullet)(x) \neq \phi_{r}(\bullet)(\mathbf{o})\right\}\right|\right]\right|<\frac{\epsilon}{4}
$$


In addition, $\lim _{n \rightarrow \infty} 2\left|\mathrm{E}_{n}\right| /\left|\mathrm{V}_{n}\right|=d$.

Now, if $\left|S_{n}\right|>\left|\mathrm{V}\left(G_{n}\right) \backslash S_{n}\right|+1$, remove the fewest number needed of the smallestdegree vertices in $S_{n}$ to obtain a bisection $S_{n}^{\prime}$, while if $\left|S_{n}\right|<\left|\mathrm{V}\left(G_{n}\right) \backslash S_{n}\right|-1$, add the fewest number needed of the smallest-degree vertices not in $S_{n}$ to obtain a bisection $S_{n}^{\prime}$. The vertices moved from one part to the other each have degree at most the median degree, which is at most twice the mean degree, $4\left|\mathrm{E}_{n}\right| /\left|\mathrm{V}_{n}\right|$, whence for large $n$, this new bisection $S_{n}^{\prime}$ satisfies

$$
\left|\frac{\operatorname{size}\left(S_{n}^{\prime}\right)}{\left|\mathrm{V}_{n}\right|}-\frac{1}{2} \mathbb{E}[|\{x \sim \mathbf{o} ; \phi(\bullet)(x) \neq \phi(\bullet)(\mathbf{o})\}|]\right|<\epsilon,
$$

which proves (4.3).

It remains to prove the asserted bounds but for MinBiFac and MaxBiFac.

Let $\sigma_{ \pm}$be the Gaussian wave functions of Csóka, Gerencsér, Harangi, and Virág (2015) with eigenvalues $\pm \rho_{d}$ for the transition operator. These are weak* limits of FIID processes, and thus so are $\operatorname{sgn} \sigma_{ \pm}$. (The two wave functions are also related to each other via the distributional equality $\sigma_{+} \stackrel{\mathcal{D}}{=} f_{*} \sigma_{-}$, where $f: \mathbb{R}^{\vee} \rightarrow \mathbb{R}^{\vee}$ is the map $(f(\omega))(x)=$ $(-1)^{d(\mathbf{o}, x)} \omega(x)$.) Consider the bisections $\left\{x \in \mathrm{V} ; \sigma_{ \pm}(x)>0\right\}$. Now for jointly normal centered random variables $\left(Z_{1}, Z_{2}\right)$, we have

$$
\mathbb{P}\left[\operatorname{sgn} Z_{1} \neq \operatorname{sgn} Z_{2}\right]=\mathbb{P}\left[Z_{1} Z_{2}<0\right]=\frac{1}{\pi} \arccos \operatorname{Corr}\left(Z_{1}, Z_{2}\right) .
$$

Since Corr $\left(\sigma_{ \pm}(x), \sigma_{ \pm}(y)\right)= \pm \rho_{d}$ for neighbors $x$ and $y$, we obtain that

$$
\operatorname{MinBiFac} \leq \frac{d}{2 \pi} \arccos \rho_{d}
$$

and

$$
\operatorname{MaxBiFac} \geq \frac{d}{2 \pi} \arccos \left(-\rho_{d}\right)
$$
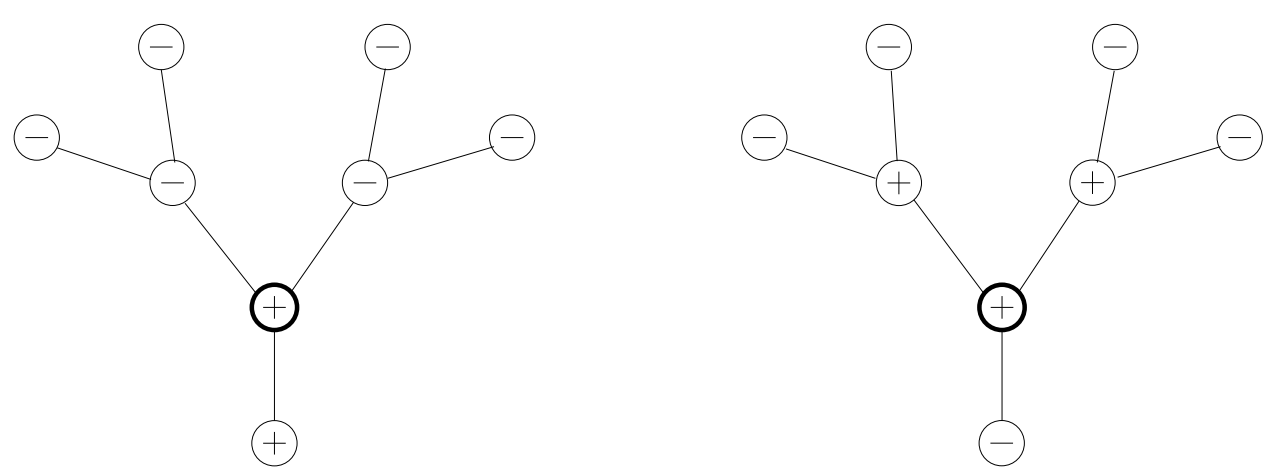

Figure 1. 
Local improvements lead to strict inequalities. That is, consider a vertex $x$ such as the heavily circled one in Figure 1, where the left figure applies to sgn $\sigma_{+}$and the right figure to $\operatorname{sgn} \sigma_{-}$. Only the case of $d=3$ is drawn, but all degrees are similar. It is easily checked that such configurations have positive probability by using the Markov property established in the proof of Theorem 3 of Csóka, Gerencsér, Harangi, and Virág (2015). When such a configuration occurs, change the value at $x$ to its opposite; likewise for configurations that are all opposite to those drawn. Note that the lower neighbor of $x$ may change as well, but the upper neighbors of $x$ will not. Thus, the number of edges incident to $x$ with the opposite sign strictly decreases on the left and strictly increases on the right.

Finally, to prove the last inequalities in (4.1) and (4.2) that involve an estimate of the arccos function, a little algebra reveals that they are equivalent to the inequality

$$
\sin \frac{2}{\sqrt{d}}<\frac{2 \sqrt{d-1}}{d}
$$

for $d \geq 3$. Substituting $x:=2 / \sqrt{d}$ shows that this is the same as $\sin x<x\left(1-x^{2} / 4\right)^{1 / 2}$. Indeed, $\sin x<x-x^{3} / 6+x^{5} / 120$ for $0<x^{2}<6$, whereas $x\left(1-x^{2} / 4\right)^{1 / 2}>x\left(1-x^{2} / 7\right)$ for $0<x^{2}<7 / 4$, and this leads to the desired inequality.

Finally, we improve Kardoš, Král', and Volec (2012), who showed that if $G$ is a finite graph of maximum degree 3 and girth at least 637,789, then there is a probability measure on edge cuts of $G$ such that each edge belongs to a random cut with probability at least 0.88672 , whence (by taking expected size of edge cuts) $G$ contains an edge cut of cardinality at least $0.88672|\mathrm{E}(G)|$. For numerical comparison, note that this translates to the following result when $G$ is 3-regular: 3-regular $n$-vertex graphs of girth tending to infinity possess subsets $S$ such that $|E(S, \vee \backslash S)| \geq(1.33008-o(1)) n$ for even $n \rightarrow \infty$. Theorem 4.1 already improved this by increasing the constant and by requiring $S$ to be a bisection.

THEOREM 4.2. If $G$ is a finite graph of maximum degree $d$ and girth at least $2 n+1$, then there is a random edge cut $\Pi$ of $G$ such that

$$
\mathbb{P}[e \in \Pi] \geq \frac{1}{\pi} \arccos \frac{-\rho_{d}}{1+\frac{d-1}{d(n-1)}}
$$

for all $e \in \mathrm{E}(G)$.

For $d=3$, this says, e.g., that if $G$ has girth at least 655 , then there is a random edge cut $\Pi$ such that $\mathbb{P}[e \in \Pi] \geq 0.89$ for all $e \in \mathrm{E}(G)$. 
Proof. It suffices to prove the analogous result on $\mathbb{T}_{d}$ via a block FIID of radius $n$ : We can then adjoin trees to $G$ in order to create a (possibly infinite) $d$-regular graph $G^{\prime}$. The block FIID can be applied to $G^{\prime}$ to obtain a random cut $\Pi^{\prime}$ of $G^{\prime}$; then we may let $\Pi:=\Pi^{\prime} \cap \mathrm{E}(G)$.

To this end, let $\mathfrak{m}$ be standard Gaussian measure on $\mathbb{R}$. Then the coordinate projections $Z_{x}:\left(\mathbb{R}^{\vee\left(\mathbb{T}_{d}\right)}, \mathfrak{m}^{\vee\left(\mathbb{T}_{d}\right)}\right) \rightarrow \mathbb{R}$ are independent standard normal random variables for $x \in \mathrm{V}\left(\mathbb{T}_{d}\right)$. Put

$$
F:=\frac{1}{\sqrt{1+(n-1) d /(d-1)}} \sum_{d(\mathbf{o}, x)<n} \frac{Z_{x}}{(-\sqrt{d-1}) d(\mathbf{o}, x)} .
$$

Then $F$ defines a block FIID $\sigma$ of radius $n$ with single marginal equal to standard Gaussian. Since

$$
\operatorname{Corr}(\sigma(x), \sigma(y))=-\frac{1}{1+\frac{(n-1) d}{d-1}} \sum_{k=0}^{n-2} \frac{2(d-1)^{k}}{\sqrt{d-1}^{2 k+1}}=-\frac{\rho_{d}}{1+\frac{d-1}{d(n-1)}}
$$

for $x \sim y$, the result follows by (4.4).

Acknowledgement. I thank Allan Sly and Yuval Peres for allowing me to include Theorem 3.1. I also thank Lewis Bowen, Yuval Peres and Jeff Steif for various discussions of this material. I am grateful to a referee for helpful remarks.

\section{REFERENCES}

ABÉRT, M. and Weiss, B. (2013). Bernoulli actions are weakly contained in any free action. Ergodic Theory Dynam. Systems, 33(2), 323-333.

Adams, S. (1992). Very weak Bernoulli for amenable groups. Israel J. Math., 78(2-3), $145-176$.

Alon, N. (1997). On the edge-expansion of graphs. Combin. Probab. Comput., 6(2), $145-152$.

Angel, O., Benjamini, I., Gurel-Gurevich, O., Meyerovitch, T., and Peled, R. (2012). Stationary map coloring. Ann. Inst. Henri Poincaré Probab. Stat., 48(2), $327-342$.

Backhausz, Á., Szegedy, B., and VirÁG, B. (2015). Ramanujan graphings and correlation decay in local algorithms. Random Structures Algorithms, 47(3), 424-435.

BALL, K. (2005a). Factors of independent and identically distributed processes with nonamenable group actions. Ergodic Theory Dynam. Systems, 25(3), 711-730.

Ball, K. (2005b). Poisson thinning by monotone factors. Electron. Comm. Probab., 10, 60-69 (electronic).

Bollobás, B. (1988). The isoperimetric number of random regular graphs. European J. Combin., 9(3), 241-244.

Bowen, L.P. (2010). A measure-conjugacy invariant for free group actions. Ann. of Math. (2), 171(2), 1387-1400. 
Bowen, L.P. (2013). Personal communication.

Burton, R.M., Denker, M., and Smorodinsky, M. (1996). Finite state bilaterally deterministic strongly mixing processes. Israel J. Math., 95, 115-133.

Burton, R.M. and Steif, J.E. (1997). Coupling surfaces and weak Bernoulli in one and higher dimensions. Adv. Math., 132(1), 1-23.

Chatterjee, S., Peled, R., Peres, Y., and Romik, D. (2010). Gravitational allocation to Poisson points. Ann. of Math. (2), 172(1), 617-671.

Chifan, I. and IoAna, A. (2010). Ergodic subequivalence relations induced by a Bernoulli action. Geom. Funct. Anal., 20(1), 53-67.

Conley, C.T. (2013). Brooks' theorem for Bernoulli shifts. Preprint, available at http: //www.math.cmu.edu/ clintonc/onlinepapers/bernoullibrooks.pdf.

Csóka, E., Gerencsér, B., Harangi, V., and Virág, B. (2015). Invariant Gaussian processes and independent sets on regular graphs of large girth. Random Structures Algorithms, 47(2), 284-303.

CsókA, E. and Lippner, G. (2012). Invariant random matchings in Cayley graphs. Preprint, http://www.arxiv.org/abs/1211.2374.

Dembo, A., Montanari, A., and Sen, S. (2015). Extremal cuts of sparse random graphs. Preprint, http://www.arxiv.org/abs/1503.03923.

Díaz, J., Do, N., Serna, M.J., and Wormald, N.C. (2003). Bounds on the max and min bisection of random cubic and random 4-regular graphs. Theoret. Comput. Sci., 307(3), 531-547. Selected papers in honor of Lawrence Harper.

Díaz, J., Serna, M.J., and Wormald, N.C. (2007). Bounds on the bisection width for random $d$-regular graphs. Theoret. Comput. Sci., 382(2), 120-130.

Evans, W., Kenyon, C., Peres, Y., and Schulman, L.J. (2000). Broadcasting on trees and the Ising model. Ann. Appl. Probab., 10(2), 410-433.

Gamarnik, D. and Sudan, M. (2013). Limits of local algorithms over sparse random graphs. Preprint, http://www.arxiv.org/abs/1304.1831.

Gurel-Gurevich, O. and Peled, R. (2013). Poisson thickening. Israel J. Math., 196(1), $215-234$.

HARAngI, V. and VirÁg, B. (2015). Independence ratio and random eigenvectors in transitive graphs. Ann. Probab., 43(5), 2810-2840.

Holroyd, A.E. (2011). Geometric properties of Poisson matchings. Probab. Theory Related Fields, 150(3-4), 511-527.

Holroyd, A.E., Lyons, R., and Soo, T. (2011). Poisson splitting by factors. Ann. Probab., 39(5), 1938-1982.

Holroyd, A.E., Pemantle, R., Peres, Y., and Schramm, O. (2009). Poisson matching. Ann. Inst. Henri Poincaré Probab. Stat., 45(1), 266-287.

Holroyd, A.E. and Peres, Y. (2003). Trees and matchings from point processes. Electron. Comm. Probab., 8, 17-27 (electronic).

HoudAYER, C. (2012). Invariant percolation and measured theory of nonamenable groups [after Gaboriau-Lyons, Ioana, Epstein]. Astérisque, 348, Exp. No. 1039, ix, 339-374. Séminaire Bourbaki: Vol. 2010/2011. Exposés 1027-1042.

Kalikow, S.A. (1982). T, $T^{-1}$ transformation is not loosely Bernoulli. Ann. of Math. (2), 115(2), 393-409. 
Kardoš, F., Král', D., and Volec, J. (2012). Maximum edge-cuts in cubic graphs with large girth and in random cubic graphs. Random Structures Algorithms, 41(4), $506-520$.

Kesten, H. (1959). Symmetric random walks on groups. Trans. Amer. Math. Soc., 92, $336-354$.

Kolmogorov, A.N. (1958). A new metric invariant of transient dynamical systems and automorphisms in Lebesgue spaces. Dokl. Akad. Nauk SSSR (N.S.), 119, 861-864.

Kolmogorov, A.N. (1959). Entropy per unit time as a metric invariant of automorphisms. Dokl. Akad. Nauk SSSR, 124, 754-755.

Kun, G. (2013). Expanders have a spanning Lipschitz subgraph with large girth. Preprint, http://www . arxiv.org/abs/1303.4982.

Lyons, R. (2013). Fixed price of groups and percolation. Ergodic Theory Dynam. Systems, 33(1), 183-185.

Lyons, R. and Nazarov, F. (2011). Perfect matchings as IID factors on non-amenable groups. European J. Combin., 32(7), 1115-1125.

Mester, P. (2011). A factor of i.i.d with uniform marginals and infinite clusters spanned by equal labels. Preprint, http://www.arxiv.org/abs/1111.3067.

Monien, B. and Preis, R. (2001). Upper bounds on the bisection width of 3- and 4regular graphs. In Sgall, Jiříand Pultr, A. and Kolman, P., editors, Proceedings of the 26th International Symposium (MFCS 2001) held in Mariánské Láznĕ, August 27-31, 2001, volume 2136 of Lecture Notes in Comput. Sci., pages 524-536. Springer, Berlin.

Ornstein, D.S. (1970a). Bernoulli shifts with the same entropy are isomorphic. Advances in Math., 4, 337-352 (1970).

Ornstein, D.S. (1970b). Factors of Bernoulli shifts are Bernoulli shifts. Advances in Math., 5, 349-364 (1970).

ORnstein, D.S. (1973). An example of a Kolmogorov automorphism that is not a Bernoulli shift. Advances in Math., 10, 49-62.

Ornstein, D.S. (1974). Ergodic Theory, Randomness, and Dynamical Systems. Yale University Press, New Haven, Conn. James K. Whittemore Lectures in Mathematics given at Yale University, Yale Mathematical Monographs, No. 5.

Ornstein, D.S. and Weiss, B. (1975). Every transformation is bilaterally deterministic. Israel J. Math., 21(2-3), 154-158. Conference on Ergodic Theory and Topological Dynamics (Kibbutz Lavi, 1974).

ORnstein, D.S. and Weiss, B. (1987). Entropy and isomorphism theorems for actions of amenable groups. J. Analyse Math., 48, 1-141.

Pemantle, R. (1992). Automorphism invariant measures on trees. Ann. Probab., 20(3), $1549-1566$.

PopA, S. (2006). Some computations of 1-cohomology groups and construction of nonorbit-equivalent actions. J. Inst. Math. Jussieu, 5(2), 309-332.

Quas, A. and Soo, T. (2016). A monotone Sinai theorem. Ann. Probab., 44(1), 107-130.

Rohlin, V.A. and Sina ̌̆, JA.G. (1961). The structure and properties of invariant measurable partitions. Dokl. Akad. Nauk SSSR, 141, 1038-1041. 
SinA $\breve{,}$ JA. (1959). On the concept of entropy for a dynamic system. Dokl. Akad. Nauk SSSR, 124, 768-771.

SLY, A. (2009). Personal communication.

Smorodinsky, M. (1971). A partition on a Bernoulli shift which is not weakly Bernoulli. Math. Systems Theory, 5, 201-203.

Soo, T. (2010). Translation-equivariant matchings of coin flips on $\mathbb{Z}^{d}$. Adv. in Appl. Probab., 42(1), 69-82.

Timár, Á. (2004). Tree and grid factors for general point processes. Electron. Comm. Probab., 9, 53-59 (electronic).

Timár, Á. (2011). Invariant colorings of random planar maps. Ergodic Theory Dynam. Systems, 31(2), 549-562.

Department of Mathematics, 831 E 3Rd St, Indiana University, Bloomington, in 47405-7106 rdlyons@indiana.edu

http://mypage.iu.edu/ ${ }^{\sim}$ rdlyons/ 